\section{LA MILITANCIA FAMILIAR COMO FORMA DE COMPROMISO POLÍTICO: EL LIDERAZGO DE LAS HERMANAS GARCÍA PÉREZ (1918-1931)}

\author{
Marta del Moral Vargas \\ Universidad Complutense \\ ORCID iD: https://orcid.org/0000-0001-9584-801X \\ mmoralva@ucm.es
}

\section{FAMILY-BASED ACTIVISM AS A FORM OF POLITICAL COMMITMENT: THE LEADERSHIP OF THE GARCÍA PÉREZ SISTERS (1918-1931)}

Cómo citar este artículo/Citation: Moral Vargas, M. del (2020). La militancia familiar como forma de compromiso político: el liderazgo de las hermanas García Pérez (1918-1931). Arbor, 196 (796): a550. https://doi.org/10.3989/arbor.2020.796n2003

Recibido: 26 mayo 2018. Aceptado: 23 octubre 2019.
RESUMEN: Este artículo aborda la trayectoria política de las hermanas Claudina y Luz García Pérez en la Agrupación Femenina Socialista de Madrid y en varios sindicatos vinculados a la Casa del Pueblo entre 1918 y 1931. Sus biografías sirven para cuestionar el estereotipo del varón activista sindical y político, al evidenciar la importancia de la militancia familiar en la adopción y mantenimiento de un compromiso de por vida con el socialismo. Además, su trasgresión a los roles hegemónicos de género pone de manifiesto el papel desempeñado por los espacios de sociabilidad política exclusivamente femeninos en la formación de mujeres líderes en regímenes que no reconocían derechos de ciudadanía a las mujeres.

PALABRAS CLAVE: Luz García Pérez; Claudina García Pérez; Agrupación Femenina Socialista de Madrid; Partido Socialista Obrero Español; relaciones de género; liderazgo; Madrid; militancia familiar.
Copyright: (C) 2020 CSIC. Este es un artículo de acceso abierto distribuido bajo los términos de la licencia de uso y distribución Creative Commons Reconocimiento 4.0 Internacional (CC BY 4.0).
ABSTRACT: This article focuses on the political biographies of two sisters, Claudina and Luz García Pérez, between 1918 and 1931 within the Agrupación Femenina Socialista de Madrid (Women's Socialist Group of Madrid) and various trade unions linked to the Casa del Pueblo. Their biographies question the stereotype of the male union and political leader by highlighting the importance of the family unit in adopting and maintaining a lifelong commitment to the socialist cause. Moreover, their transgression of the hegemonic gender system highlights the role played by women-only political fora in relation to the formation of female political leaders within regimes in which women were deprived of the right to vote.

KEYWORDS: Luz García Pérez; Claudina García Pérez; Agrupación Femenina Socialista de Madrid; Partido Socialista Obrero Español; gender; leadership; Madrid; family-based activism. 
“iSólo tres mujeres!”, exclamó Claudina García Pérez en su primera contribución a El Socialista con motivo de la celebración del XI Congreso del Partido Socialista (PSOE), y unos cinco meses después de haber ingresado en su agrupación femenina de Madrid, en julio de 1918. En su artículo, publicado en la primera plana del diario, se preguntaba: "¿es que no hay mujeres que sientan deseos de rebelarse en contra de los opresores, cuando con nosotras son precisamente más injustos y somos las más oprimidas en todos los órdenes sociales y económicos?". Sus propias compañeras le respondían con rotundidad que "hay pocas entre nosotras que sepan ocuparse de estos asuntos y, claro, solo se nombra como delegados a los hombres porque saben más". En este texto Claudina formuló su compromiso político con el partido al que acababa de afiliarse al manifestar que la redención de las mujeres debía corresponderles a ellas mismas y añadía:

Es preciso irnos acostumbrando a saber pedir las cosas, a defender los derechos que en justicia debemos tener; es necesario ser fuertes para imponernos $y$, en una palabra, para ayudar a los hombres en esta lucha titánica del ideal que nace, y de las viejas costumbres que mueren porque ya no tienen razón de ser (García Pérez, 1918, 28 de noviembre).

Desde su ingreso en la Agrupación Femenina Socialista de Madrid (AFSM) en 1918, Claudina y Luz García Pérez asumieron un compromiso con el socialismo que mantuvieron durante toda su vida. Se formaron como líderes políticas en la AFSM, donde ocuparon varios cargos de responsabilidad y coincidieron con otras grandes figuras del partido como Virginia González Polo. Desde 1927, al disolverse la AFSM, se dedicaron a promover el sindicalismo femenino. A partir de los años treinta, alcanzaron puestos destacados en la Unión General de Trabajadores (UGT) y fueron propuestas como candidatas en las listas del PSOE en los comicios generales de noviembre de 1933. Las dos se vieron obligadas a exiliarse en México debido a la guerra civil, y allí continuaron manteniendo vivo su compromiso a través de la fundación de la Agrupación de Socialistas Españoles (Sección México) en 1952 (Martín-Nájera, 2010).

Sin embargo, su trayectoria pública, política y sindical, como la de sus compañeros varones, no puede entenderse sin considerar la dimensión privada, familiar, de ambas líderes. Como sucedía en otras culturas políticas como los republicanismos, la militancia de la unidad familiar era clave en el mantenimiento del compromiso adquirido (Sanfeliu Gimeno, 2005a; Sanfeliu Gimeno, 2014). El matrimonio de Claudina García
Pérez con Modesto Pereira Sanz, miembro de la Sociedad de Ebanistas de Madrid, afiliado a la Agrupación Socialista Madrileña (ASM) y tesorero de la Mutualidad Obrera de la Casa del Pueblo, alumbró un hogar de socialistas comprometidos con el proyecto político del PSOE. Esta unidad familiar, representativa de muchas otras vinculadas a la Casa del Pueblo, cuestiona la imagen estereotipada del varón activista sindical y político que presupone la desvinculación de las mujeres de una práctica de militancia proactiva. Sostengo que en la España del primer tercio del siglo XX adquirir y mantener un compromiso con un proyecto político transgresor con el orden hegemónico dio como resultado, en muchos casos, una militancia de carácter familiar. La apuesta por construir una nueva sociedad basada en el ideal socialista, planteaba un desafío al sistema del turnismo corrupto de la Restauración. El respaldo familiar en esta lucha política se convertía en la pieza necesaria frente a los altos costes emocionales del activismo cotidiano (Hernando Gonzalo, 2012). En muchos casos, más que empatía con las tribulaciones del titular del único carné de afiliado, implicaba la adopción de un compromiso igual de varios miembros de la familia, hombres y mujeres.

Además, la trasgresión representada por quienes defendían estos planteamientos se duplicaba en el caso de las mujeres, al invadir un espacio público asignado a los varones. En este sentido, cobra relieve la AFSM, como espacio de sociabilidad política exclusivamente femenino, donde las trabajadoras podían definir su propio proyecto reivindicativo lejos de la censura masculina general y de la expresada por algunos de los varones vinculados a la Casa del Pueblo (Moral Vargas, 2012, pp. 145-166). Tanto Luz como Claudina García se erigieron como líderes capaces de desafiar ese entorno profundamente machista, con el que convivieron tanto dentro como fuera de la sede socialista de la calle Piamonte.

\section{LA MILITANCIA FAMILIAR EN EL SOCIALISMO MADRILEÑO}

Claudina y Luz García Pérez formaron parte de la incesante corriente migratoria que venía impulsando el crecimiento de Madrid, casi sin interrupción, desde que se convirtió en capital. Claudina llegó a Madrid hacia 1909 , con unos veinte años, y su hermana Luz la seguiría unos meses después, a la edad de quince años. Procedían de Miranda de Ebro, Burgos, donde nacieron en 1889 y 1895, respectivamente. ${ }^{1}$ Ambas trabajaban como obreras de la aguja a destajo y a domicilio, uno de los sectores profesionales en el que se 
empleaba un porcentaje significativo de la población activa femenina de Madrid a principios de siglo y también uno de los peor remunerados. Según las estadísticas del Instituto de Reformas Sociales, entre 1914 y 1925 las industrias del vestido en la ciudad de Madrid daban trabajo a unos 3.000 hombres y 9.000 mujeres (Ministerio de Trabajo, Comercio e Industria, 1927, p. 186). Estas cifras han de considerarse con cautela debido al alto porcentaje de obreras de la aguja que formaban parte de la economía sumergida de la capital, sin ver su labor reflejada en las estadísticas oficiales (Capel Martínez, 1986, pp. 116-196; Nash, 1996; Nielfa Cristóbal, 1999, pp. 158-159; Núñez Orgaz, 1989).

Este era el caso de las hermanas García Pérez durante sus primeros años de residencia en la capital. Las condiciones de trabajo más duras del sector eran precisamente las vividas por quienes se empleaban a domicilio y a destajo. Al no estar concentradas en un taller, no eran candidatas a la visita de la inspección, aun con su alcance limitado en la práctica (Rey Reguillo, 1992, pp. 349-350), ni a las mejoras higiénicas y sanitarias introducidas progresivamente en los talleres. Desarrollaban su labor en la propia vivienda o en la del oficial u oficiala, generalmente cuartos sin ventilación ni luz natural, hasta completar el encargo por el que recibían un pago acordado previamente, independientemente de las horas que tuvieran que invertir para ello. Además, sus jornales, ya de por sí reducidos, eran inferiores a los percibidos por los trabajadores varones. Las diferencias salariales entre hombres y mujeres entre 1908 y 1920 en la provincia de Madrid, oscilaron entre 1,08 y 3,75 pesetas. Hacia 1914 , las bordadoras percibían entre 0,75 y 2,50 pesetas de jornal según su categoría (Núñez Orgaz, 1989). En el padrón de 1925, tanto Claudina como Luz se inscribieron como bordadoras oficialas, después de años declarando dedicarse a sus labores o manifestando no ejercer profesión alguna.

Esta situación de desprotección laboral, sin embargo, no animó a las hermanas García a afiliarse a ninguna sociedad de resistencia ni agrupación política durante sus primeros nueve años en Madrid, a pesar de contar con varias opciones. De hecho, la década de los años diez destaca por el dinamismo asociativo de distinto signo ideológico protagonizado por las trabajadoras de la capital. Sus primeros meses de residencia en la ciudad coincidieron con la fundación del Sindicato Obrero Femenino de la Inmaculada. Esta asociación católica patrocinada por María de Echarri y el párroco Juan José Santander fue fundada el 14 de noviembre de 1909. Su vocación era servir de antídoto frente al auge del asociacionismo de clase entre las obreras. Se dirigía, además, de forma específica a las trabajadoras de la aguja por constituir uno de los sectores profesionales femeninos más numerosos (Arce Pinedo, 2008, pp. 31-95; Capel Martínez, 1980; García Basauri, 1979; García Basauri, 1982, p. 19; Moral Vargas, 2012, pp. 120-129). Según el Instituto de Reformas Sociales, hacia 1918 contaba ya con unas 700 afiliadas (Instituto de Reformas Sociales, 1918, p. 301). Tras los sucesos de la Semana Trágica de 1909, donde las mujeres habían participado activamente en la protesta, los representantes del catolicismo social en Madrid reaccionaron contra la creciente presencia pública de agrupaciones políticas como la AFSM, fundada el 25 de marzo de 1906, y varios sindicatos de clase.

Entre estos últimos destacan la Sociedad de Profesiones y Oficios Varios, fundada el 1 de junio de 1894 y vinculada a la UGT desde 1908. Según los datos conservados para el periodo 1911-1914, la asociación contó con 4.447 personas afiliadas, de las cuales 1.001 eran mujeres. En diciembre de 1914, figuraban de alta en la asociación 2.154 personas, de las cuales 419 eran mujeres, un $20 \%$ sobre el total. De ellas, al menos 42 declaraban ejercer un oficio de la aguja. Finalmente, hay que mencionar la Sociedad de Modistas de Madrid, con sede en la calle Mayor 1, fundada el 19 de junio de 1904, con 352 afiliadas. Esta asociación mantuvo un estrecho vínculo con la AFSM a través de su secretaria, Otilia Solera García. La AFSM, gracias a la mediación de Solera, entre otras, trató sin éxito de refundar la Sociedad de Modistas como una Varia femenina, en 1913 (Moral Vargas, 2013, pp. 6-18).

Sin embargo, hubieron de transcurrir nueve años, los mismos que tardaron en trasladarse al piso principal de la calle Santa Bárbara 6, para que Claudina, Luz y la hermana pequeña de estas, recién llegada a Madrid, M. a Concepción García Pérez, se dieran de alta en la AFSM con fecha de 1 de junio de 1918 (M.a Concepción y Luz) y de 1 de julio del mismo año (Claudina). Pero algo más había cambiado en la vida de las hermanas García Pérez: el contrato de inquilinato de este nuevo piso data precisamente del 10 de junio de 1918 y fue firmado por Modesto Pereira Sanz, que en el padrón de 1920 ya figura como cabeza de familia y esposo de Claudina García Pérez. Modesto y Claudina debieron de contraer matrimonio en torno a la primera mitad de 1918 y se establecieron en el piso principal del inmueble donde habían residido Claudina y Luz hasta entonces. Además, ahora la familia crecía con la llegada de M. a Concepción y de su madre, Leocadia Pérez Román. 
El matrimonio de Claudina con Modesto Pereira Sanz inició no solo un compromiso de por vida entre los cónyuges, sino también un compromiso de por vida para las hermanas García Pérez con el socialismo. Modesto Pereira era de profesión ebanista y pertenecía a la Sociedad de Ebanistas de Madrid (UGT), con el carnet no 8, así como a la ASM, con el carnet no 144. Desde 1916, trabajaba como cajero-tesorero de la Mutualidad Obrera de la Casa del Pueblo, como declaraba en el padrón municipal de 1920. Además, a la altura de 1918 ya había representado a varias sociedades de oficio en los congresos XI y XIII de la UGT, en 1914 y 1918, respectivamente (Martín-Nájera, 2010). Se trataba, por lo tanto, de un militante abiertamente comprometido con el proyecto socialista. Podríamos afirmar que el matrimonio de Claudina y Modesto dio como resultado la extensión del compromiso político con la causa socialista a la nueva familia extensa resultante de esta unión. Las tres hermanas se dieron de alta como afiliadas en la AFSM y dos de ellas se convertirán en importantes líderes sindicales y políticas en las décadas siguientes. Leocadia Pérez se incorporó a la directiva de la AFSM como contadora en 1923.

No fue el único caso dentro del socialismo madrileño. Sabemos que al menos 138 afiliadas a la AFSM estaban emparentadas con militantes socialistas, tanto de la agrupación local (ASM) como de los sindicatos y otras agrupaciones de la Casa del Pueblo (véase figura 1). Lamentablemente, carecemos de datos para los familiares de las restantes 446 afiliadas a la AFSM. En todo caso, que no dispongamos de los datos no desmiente necesariamente que pertenecieran a familias con más de una persona vinculada a las sociedades obreras socialistas en Madrid. Esas 138 familias confirman que la militancia familiar constituyó una forma de compromiso habitual entre aquellas personas vinculadas al ideal socialista. Es el caso de líderes como Virginia González Polo, casada con Lorenzo Rodríguez, afiliado a la ASM y a la Sociedad de Zapateros y su hijo César, afiliado a la Juventud Socialista de Madrid (JSM) y a la ASM, hasta la escisión de 1921; Juana Taboada, afiliada a la Sociedad de Propaganda desde 1904 y a la AFSM desde su fundación, estaba casada con Jacobo Castro, tesorero de la ASM entre 1907 y 1936 y miembro de la Sección de Propaganda desde 1908; Mariano Medel, compañero de Matilde Otero, fundadora de la AFSM, estaba afiliado a la Sociedad de Obreros Carpinteros, a la ASM y a la Sección de Propaganda. Además, como el marido de Claudina García, trabajó para la Mutualidad Obrera, en su caso como secretario, y perteneció a las directivas de Escuelas Laicas Graduadas y de la Sociedad de Profesiones y Oficios Varios. También se repite el patrón entre afiliadas con menor presencia en los cuadros directivos, como Josefa Iglesias, casada con Manuel Cordero, el miembro del PSOE que demostró el compromiso más firme con la defensa de los derechos políticos de las mujeres en las Constituyentes de 1931, como relataba Clara Campoamor en sus memorias (Campoamor, 1936/2001, p. 103). Sin duda, se trató de una muestra más de coherencia del compromiso político de la familia Iglesias-Cordero con la militancia femenina en el socialismo y no fue la única (Moral Vargas, 2012, p. 367, p. 374, p. 404). Podríamos continuar citando ejemplos hasta repasar las 138 familias sobre las cuales se conservan datos contrastados del compromiso socialista de varios de sus integrantes (Martín-Nájera, 2010; Moral Vargas, 2012).

Figura 1. Número de afiliadas a la AFSM con relación de parentesco con militantes socialistas (1906-1927)

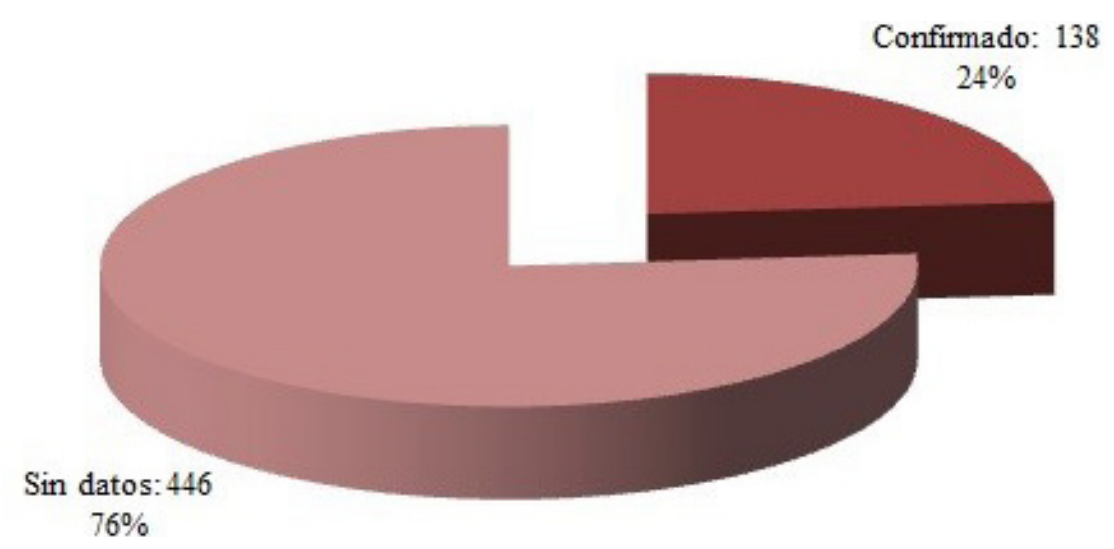

Fuente: elaboración propia a partir de AFSM, Registro de asociadas, 1906/1927. Fundación Pablo Iglesias (FPI), Archivo y Biblioteca, y AVM. Padrones Municipales de Habitantes, Madrid, 1905, 1910, 1915, 1920, 1925, 1930. 
Como ya se afirmaba en la introducción, esta era también una práctica común entre la militancia republicana desde décadas atrás. Las Damas Rojas de Barcelona, por ejemplo, eran descritas en absoluta comunidad de pensamiento con sus maridos o padres, comentando entre todos las novedades políticas recogidas por la prensa (Ullman, 1972, pp. 159-161). En Valencia, se organizaban diversos actos públicos para toda la familia, con el fin de transformar los lazos de parentesco en ideológicos, además de emocionales (Sanfeliu Gimeno, 2005a, pp. 135-178; Sanfeliu Gimeno, 2005b). Entre los librepensadores y masones también se han trazado vínculos ideológicos compartidos entre generaciones o entre cónyuges. Por ejemplo, el matrimonio librepensador integrado por Belén Sárraga y Emilio Ferrero o el masón, formado por Natividad López, simbólico Juana de Arco, y Miguel Polo (Ortiz Albear, 2005, p. 103; Ramos Palomo, 1998, p. 84). La proximidad de muchos de los planteamientos de estas culturas políticas también dio lugar a familias cuyo compromiso hizo convivir varias de ellas o incluso experimentó una evolución. En Madrid, un ejemplo elocuente lo constituye la familia de la ya citada líder sindicalista y (sucesivamente) republicana, socialista y comunista, Otilia Solera, iniciada en la logia Ibérica, en 1907. El compromiso de los Solera quedó labrado para la posteridad en la lápida de la sepultura familiar del Cementerio Civil de Madrid, por encargo de la propia Otilia (véase figura 2) (Moral Vargas, 2012, p. 104; Ortiz Albear, 2005, p. 108). Además, esa militancia familiar no era exclusiva de las culturas políticas progresistas. El carlismo, por ejemplo, también se nutría del respaldo familiar y la trasmisión del ideal entre generaciones. Las mujeres, sin embargo, quedaron relegadas al espacio privado hasta que el efecto combinado de la apertura del marco de oportunidades políticas que trajeron consigo las primeras décadas del siglo y la modernización en relación con la construcción de una sociedad de masas dieron lugar a la conformación de su propia agrupación segregada, las Margaritas (Canal, 1997; Miralles Climent, 2005, pp. 170-175).

En definitiva, durante estos años el compromiso político se formulaba y reforzaba en muchas ocasiones a través del vínculo emocional constituido por la familia, especialmente en el seno de aquellas culturas políticas situadas en los márgenes del sistema de la Restauración. Ese vínculo emocional se completaba a través de la integración de cada uno de los miembros del núcleo familiar en sus respectivos grupos de pares en función de su sexo, edad y actividad profesional (grupos de jóvenes, de mujeres, sociedades de oficio, etc.). Los altos costes emocionales experimentados por quienes desa-
Figura 2. Lápida de la sepultura de la familia Solera. Cementerio Civil de Madrid

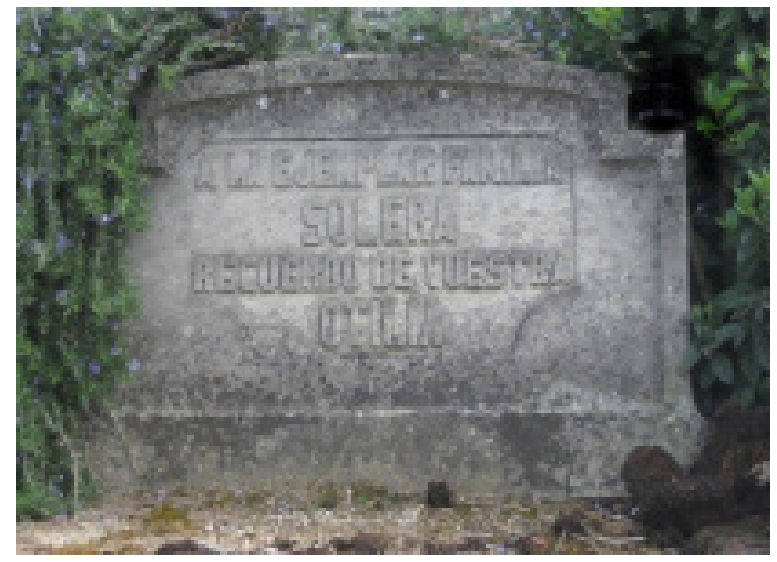

Fuente: fotografía tomada por Marta del Moral Vargas, 2009.

fiaban el orden social y político hegemónico se sobrellevaban mejor con el apoyo tanto del núcleo familiar como de los grupos de pares que alimentaban un sentimiento de comunidad, de pertenencia a un colectivo que luchaba por una meta común, una identidad de la que hombres, mujeres, niños y niñas eran partícipes (Hernando Gonzalo, 2012). En ese marco ha de entenderse el conjunto de las iniciativas políticas, sociales y culturales de estas culturas políticas. Es decir, en el caso por ejemplo del socialismo madrileño, no tiene sentido otorgar importancia preeminente al partido y a algunas de sus sociedades obreras para tratar de sumergirnos en el día a día de la militancia. Entender el compromiso político socialista pasa por concebirlo como una forma de vida en su conjunto, sin definir compartimentos estancos. El mantenimiento de dicho compromiso cobra sentido completo al valorar su dimensión cotidiana, la que implicaba a toda la familia en las agrupaciones políticas (AFSM, ASM y Juventud Socialista, Sección de Propaganda) y en las sociedades de oficio, pero también en la Mutualidad Obrera, en las Escuelas Laicas, en la Cooperativa de la Casa del Pueblo, en las meriendas en la Casa de Campo del 1 응 de mayo, en el Orfeón Socialista, en los proyectos de Casas Baratas, en los entierros en el Cementerio Civil... (Moral Sandoval, 2008). Es decir que, frente a las manifestaciones discursivas sobre el carácter, las capacidades y la posición especial y separada que las mujeres debían ocupar en las sociedades de la modernidad que inundaban libros, periódicos, conferencias y tertulias en esta época, las prácticas cotidianas de estos grupos evidencian que los límites para el ejercicio del compromiso político entre hombres y mujeres eran en realidad difusos y, en la mayoría de los casos, remaban en la misma dirección de forma intencionada. 
Sin embargo, centrándonos exclusivamente en las culturas políticas progresistas, a pesar de compartir esa vocación por incorporar a la familia a la lucha por los ideales políticos, no todas fueron igual de receptivas a la militancia pública de las mujeres en sus organizaciones. Las actitudes machistas caracterizaron probablemente a todos los grupos que defendían un modelo político progresista para el Estado, y otro tradicional y basado en la ideología de la domesticidad para la familia. Valga como ejemplo de los muchos existentes, la negativa de Melquiades Álvarez a compartir tribuna con Belén Sárraga, en 1899, aduciendo que "las mujeres no deben tomar parte en esas actividades" (Radcliff, 1994, p. 386).

Sin lugar a dudas el panorama del socialismo madrileño contaba con muchas actitudes semejantes. Pero la diferencia entre los socialistas y otros grupos estriba, por un lado, en el marco de oportunidades que se abrió mediante la creación, y sobre todo la relativa continuidad temporal, de foros exclusivamente femeninos que incluyeron la AFSM, desde 1906, y las sociedades de oficio para las trabajadoras. Esos espacios reforzaron el ejercicio de una militancia donde las mujeres ya no eran simples acompañantes en las actividades organizadas ad hoc por los varones, sino que tomaban la iniciativa en consonancia con las identidades colectivas específicas que ahora podían construir como mujeres trabajadoras libres de la injerencia masculina. Era posible, de este modo, incorporarse desde una identidad propia como mujeres socialistas -autónoma y con sus demandas propias- al compromiso político formulado en el núcleo familiar.

Además, el mantenimiento de esos espacios de militancia segregada para las mujeres en una época - las primeras décadas del siglo XX- en la que las actitudes favorables a su actividad política pública no eran mayoritarias garantizó la formación de futuras líderes femeninas que eclosionaron en las décadas de los años veinte y treinta y que, de otro modo, posiblemente habrían sido menos numerosas. Es decir, transformó la cultura política socialista, dando cabida al liderazgo femenino activo que, aunque contaba con antecedentes previos a la fundación de la primera agrupación política femenina en Bilbao en 1904 no disponía de un espacio exclusivo para ellas (Almond y Verba, 1970, p. 30). Esos espacios exclusivamente femeninos en el marco de los centros obreros constituyeron el entorno de socialización necesario para que muchas mujeres adquirieran las habilidades personales, es decir, la compe- tencia política, que les convirtió en líderes dentro del PSOE y la UGT (Natera Peral, 2001, p. 76). Entre estas líderes destacan Virginia González, las hermanas Claudina y Luz García, Juana Taboada, Carmen González, etc. Un papel similar jugaron otras asociaciones de mujeres como las Damas Rojas del Partido Republicano Radical, de donde procedieron figuras destacadas del sufragismo como Carmen de Burgos, Consuelo Álvarez, etc. Sin embargo, la pervivencia más limitada en el tiempo de este colectivo y un respaldo numérico probablemente más débil hizo que muchas de sus líderes terminaran completando su trayectoria de liderazgo en otros como la propia AFSM, Fraternidad Cívica, la Unión de Mujeres de España, la Cruzada de Mujeres Españolas, etc. (Fagoaga, 1985).

El otro factor que diferencia el microcosmos socialista de otras culturas políticas de la época tiene que ver con la extracción social de las mujeres que salieron de su cantera de liderazgo femenino, en relación con dos aspectos: su adscripción profesional (o la de sus familiares) y la militancia de uno o varios de sus familiares directos. Si bien resulta un hecho probado que en la AFSM, como en otras organizaciones socialistas europeas, militaron mujeres de variado estatus social -maestras, telegrafistas, profesoras en partos, tenedoras de libros...- y no solo trabajadoras manuales -obreras de la aguja, lavanderas, planchadoras, cigarreras, etc.- (Boxer, 2008; Moral Vargas, 2012), fueron las trabajadoras manuales (o esposas de trabajadores manuales) de destacada militancia familiar -varios de cuyos miembros estaban implicados activamente en los sindicatos y en las organizaciones del partido- las que sobresalieron como líderes. Por citar algunos ejemplos, es el caso de Virginia González Polo (guarnecedora de calzado, caracterizada más arriba); de Juana Taboada, que declaraba como profesión sus labores y estaba casada con Jacobo Castro, de oficio solador, tesorero de la ASM durante casi treinta años; Carmen Bernal, también dedicada a sus labores, casada con Manuel Guijarro, empleado municipal fundador de la Sociedad de Gas y Electricidad, afiliado a la Agrupación de Obreros y Empleados Municipales y a la ASM; Josefa Bernal, guarnecedora de calzado, casada con Bernardo Lumbreras, albañil fundador y afiliado a la Sociedad de albañiles El Trabajo, afiliado a la ASM, de la que era cobrador, y repartidor de El Socialista; Otilia Solera, modista de sombreros, hermana de un cerrajero, un guarnicionero y un platinista... y por supuesto de las hermanas García Pérez. 


\section{LAS HERMANAS GARCÍA PÉREZ: DE LA AGRUPA- CIÓN FEMENINA AL LIDERAZGO SINDICAL}

Claudina y Luz García Pérez encarnan de forma paradigmática la formación en el liderazgo socialista a partir de la militancia familiar. Ambas dirigentes encontraron en la AFSM su primer espacio de adscripción política y allí se entrenaron en el aprendizaje de estrategias organizativas, en la oratoria, y sobre todo en la autoafirmación como promotoras activas de la propaganda socialista entre las trabajadoras en un contexto que desincentivaba el liderazgo de las mujeres. En 1927, ya disuelta la AFSM, lejos de desvincularse de la militancia concentraron todos sus esfuerzos en la propaganda sindical y nunca abandonaron las filas del PSOE, aun viéndose obligadas a exiliarse en México, como consecuencia del desenlace de la guerra civil. La importancia del reclutamiento familiar combinado con el fomento del liderazgo femenino fue destacada por ellas mismas en algunos de los artículos que publicaron en El Socialista. Así, en 1928, señalaban el papel que tocaba desempeñar a los varones del partido con el fin de incorporar entre sus novias, compañeras y esposas a militantes verdaderamente comprometidas para engrosar las filas del PSOE. Para las hermanas García Pérez resultaba crucial que este compromiso adquirido fuese firme y sostenido en el tiempo, y que implicara a la nueva familia:

Mucho nos satisface que haya compañeros que piensen en atraer a sus novias a las filas socialistas porque así nos aseguran que procurarán buscar compañera entre aquellas mujeres de más fácil comprensión hacia el ideal; pero tenemos que decirles que hubo compañeras de socialistas que en la época del noviazgo transigían y hasta se interesaban por las cosas de la organización; pero que una vez casadas han continuado tan reaccionarias o, por lo menos, tan indiferentes como antes, $y$ hay que suponer la lucha que entabla un hombre convencido que, además de tener que ocuparse de la organización, según le aconseja el ideal que sustenta, tropieza con la incomprensión de su compañera, que le entorpece cuanto puede, aunque sea inconscientemente, su labor de proselitismo (García Pérez y García Pérez, 1928, 11 de noviembre).

Esa labor individual de cada compañero debía completarse con el apoyo por parte de las asociaciones. Por ese motivo, defendían su formación como propagandistas en noviembre de 1928, un año después de haberse disuelto la AFSM, el único espacio para la militancia segregada de las mujeres socialistas, en Madrid:
Por eso siempre recomendamos a las Agrupaciones y a todos los compañeros que deben poner la mayor atención, el mayor cuidado en capacitar a ese puñado de mujeres socialistas, pues ellas, educadas, pueden hacer en este aspecto de la organización más que los hombres, porque hablan a las mujeres en su propio lenguaje; y la prueba está en que a las mujeres les escuchan y a los hombres ni siquiera van a oírles (García Pérez y García Pérez, 1928, 11 de noviembre).

Durante su paso por las filas de la AFSM, entre 1918 y 1927, Claudina y Luz García ocuparon diversos cargos destacados que les formaron en la adquisición de destrezas de liderazgo. Claudina, unos pocos meses después de su ingreso, fue elegida secretaria del Comité de dirección. Además, ocupó muchos otros puestos de importancia, especialmente a raíz de la escisión comunista que, como en el resto de agrupaciones políticas del PSOE, también afectó a las filas de la AFSM. Fue su presidenta entre 1921 y 1922, y de nuevo entre 1923 y 1925, y su vicepresidenta entre 19221923 y 1926-1927. Luz, por su parte, fue elegida vocal en 1920 y, tras la escisión tercerista, ocupó los cargos de tesorera (1921) y secretaria (1921-24 y 1926)2.

Su actividad política en esta primera etapa fue muy intensa, combinando la propaganda de la AFSM con la sindical. Claudina García era convocada para representar a las mujeres de la AFSM en plenos nacionales, como el de las Juventudes Socialistas en 1925, o en grandes eventos en la Casa del Pueblo, como la velada necrológica en homenaje a Pablo Iglesias, en el primer aniversario de su fallecimiento. Asimismo, se les invitaba a dirigir palabras de felicitación o de aliento a las sociedades de oficio. Por ejemplo, en el 8 o aniversario de la Sociedad de biseladores de lunas o con motivo de la negociación del convenio laboral de la Sociedad Unión Gorrera Madrileña en 1926. Además, Claudina García impartió conferencias sobre las cuestiones que centraron el debate político entre 1918 y 1927. Por ejemplo, en febrero de 1919 pronunció una conferencia titulada "Bolchevismo o socialismo de acción", en el momento álgido de apoyo del socialismo español a la revolución rusa, que comenzaría a declinar unos meses después (Meaker, 1978, pp. 151-469). Este declive, como es bien sabido, culminó con la escisión del PSOE y sus agrupaciones entre defensores de la unión al Komintern y partidarios de la reconstrucción de la Segunda Internacional (Gómez Llorente, 2004, pp. 50-56; Juliá, 1996, pp. 104-111; Meaker, 1978, pp. 450-498), incluyendo a las hermanas García Pérez. Claudina García y María Hernández, en nombre de la AFSM, como delegadas al Congreso extraordinario del PSOE de 1921, publicaron un Ilamamiento a todas las agrupacio- 
nes femeninas socialistas españolas para pedirles que expresasen su apoyo a la reconstrucción (véase Aniversario de la muerte de Iglesias, 1926, 8 de diciembre; Círculo Socialista del Sur, 1919, 7 de febrero; García y Hernández, 1921, 15 de abril; Movimiento obrero. Fiesta de aniversario. 1926, 11 de julio; Notas societarias. Reunión del pleno nacional de Juventudes Socialistas, 1925, 11 de noviembre; Reuniones y conferencias. De Madrid. Círculo Socialista del Sur, 1919, 7 de febrero).

Por otro lado, Claudina y Luz García participaron en la campaña socialista para atraer el voto de las mujeres, organizada tras conocerse la aprobación del Estatuto municipal del 8 de marzo de 1924, que otorgaba este derecho a las mujeres cabeza de familia, mayores de 23 años, no sujetas a la autoridad de ningún varón (Franco Rubio, 1981; Gómez-Ferrer y Moral Vargas, 2015, p. 41). Luz, como secretaria de la AFSM, lanzó un llamamiento a todas las AFS para organizar la movilización: "ante la extraordinaria importancia que están llamadas a tener las organizaciones femeninas en nuestro país, una vez que desde las próximas elecciones tendremos derecho a intervenir en la designación de concejales". Claudina, por su parte, impartió conferencias como la titulada "El voto femenino", en la Casa del Pueblo de Tetuán de las Victorias y rindió cuentas ante la AFSM de las actividades llevadas a cabo (véase Agrupación Femenina Socialista de Madrid, 1924, 26 de julio; La Agrupación Socialista Madrileña y el voto femenino, 1924, 24 de septiembre; Movimiento obrero. Conferencia, 1924, 20 de agosto; Presidencia del Directorio Militar. Estatuto Municipal de 8 de marzo de 1924) ${ }^{3}$.

La aproximación creciente de las socialistas a iniciativas que tenían que ver con los derechos sociales y políticos de las mujeres en los años veinte, al margen del proyecto socialista, se plasmó en la colaboración en otros actos como el mitin por la investigación de la paternidad, impulsado por la AFSM y en el que participaron su entonces presidenta, Carmen González, la propia Claudina García y otra dirigente, Julia Vega, junto a Isabel Oyarzábal, Clara Campoamor y Concepción Aleixandre (véase La investigación de la paternidad, 1926, 1 de mayo). Ya en 1919, Claudina daba cuenta de sus convicciones feministas en la prensa del PSOE: "nos damos perfecta cuenta de que en el engranaje de nuestra máquina productiva, oficial o particular, económica o política, falta un elemento considerable, que es la mujer". En este artículo denunciaba que la causa era la desigualdad de trato y educación padecida por las mujeres desde su infancia y denunciaba la necesidad de permitirles el acceso a una formación en igualdad de condiciones con la recibida por los varones, para permitir su contribución activa al progreso social. La propuesta de un "programa de reivindicaciones feministas" fue liderada por Claudina en la asamblea de la AFSM del 17 de enero de 1926. Dicho programa incluía, entre otras medidas, la eliminación del artículo 438 del Código Penal, que establecía una pena más dura para las mujeres en caso de cometer adulterio; la libre disposición de sus bienes por parte de la mujer casada; la igualdad de derechos de ambos progenitores sobre los hijos; el reconocimiento legal de los hijos habidos fuera del matrimonio, o el establecimiento de un cuerpo de funcionarias para el cuidado de los menores cuyas madres trabajaran fuera del hogar. El 18 de noviembre de 1932, Clara Campoamor en nombre de la Unión Republicana Femenina, hizo acto de entrega oficial al Congreso de los Diputados de un libro para conmemorar el reconocimiento en la Constitución de 1931 de "todos los derechos civiles y políticos a la mujer". En él, junto a las firmas de Clara Campoamor, María Martínez Sierra, Elisa Soriano, Julia Peguero de Trallero, constan las de Claudina y Luz García y otras socialistas como Hildegart Rodríguez y Elena Viñuelas, poniendo de manifiesto la incorporación de la causa feminista al compromiso socialista por parte de estas líderes. Como ya vimos en el caso de Manuel Cordero, la adquisición de tal compromiso no fue privativa de las mujeres del PSOE, sino que incluyó también a sus compañeros o esposos pasando a ser un rasgo más de su identidad política familiar (García Pérez, 1919, 20 de febrero) ${ }^{4}$.

Tras la disolución de la AFSM, en mayo de 1927, Claudina y Luz García promovieron la organización de las obreras de la aguja, sector que conocían por su propia experiencia profesional, a través de la Asociación de obreras modistas y de sombreros de señora de Madrid y sus limítrofes y la Sociedad de obreras en ropa blanca, desde 1928. Luz García integró, además, la directiva de la Federación Nacional del Vestido y el Tocado en 1929. En 1931, las hermanas García extendieron su labor a otro de los sectores con mayor presencia de población activa femenina en la capital (Nielfa Cristóbal, 1999), mediante la fundación de la Sociedad de obreros y obreras del hogar. Además, Luz García fue elegida vocal de la ASM, en 1928. Como se ha señalado más arriba, el compromiso de ambas líderes se mantuvo activo durante toda su vida.

\section{CONCLUSIONES}

La militancia familiar constituye una forma de compromiso político esencial para entender las formas de vida y activismo de ciertos sectores de las culturas políticas progresistas de la Restauración. Implicó la incorporación de todos los miembros de la unidad familiar a 
una causa política a través de lazos afectivos e ideológicos. Esos ingredientes garantizaron la firmeza de dicho compromiso y su durabilidad en el tiempo, siendo mantenido de por vida en muchos casos, como el representado por las hermanas Claudina y Luz García Pérez.

Una de las culturas políticas en que se adoptó de forma consciente fue la socialista durante el primer tercio del siglo XX. El análisis de su relevancia desafía la visión predominante en la historiografía del varón militante sindical y político. En un contexto como el del Madrid de las primeras décadas del siglo XX, la vivencia del compromiso con la causa política del socialismo implicaba en decenas de casos a los varones, a las mujeres, a los niños y a las niñas ligados a la Casa del Pueblo. Concebir la historia del socialismo en esos años teniendo en cuenta la realidad de la militancia familiar nos permite entender la emergencia de líderes femeninas destacadas en un contexto de exclusión de las mujeres de los derechos de ciudadanía. Estas líderes protagonizaron una doble transgresión a través de su actuación política. Por un lado, en relación con el modelo de género hegemónico, basado en la ideología de la domesticidad. Por otro, con el propio orden político de la Restauración cimentado en la marginación de los partidos que no integraban el siste- ma del turno. Es el caso paradigmático de Claudina y Luz García Pérez, pero también el de otras dirigentes como Virginia González Polo, Otilia Solera, Juana Taboada, etc. También resulta clave para comprender la incorporación decidida de las reivindicaciones feministas al programa socialista y su defensa frente a la campaña del miedo acerca del signo conservador que se suponía iba a caracterizar al voto femenino a partir de 1931. La defensa en las Cortes del reconocimiento de este derecho para las mujeres por parte de Manuel Cordero, casado con una afiliada a la AFSM, aporta los elementos necesarios para contextualizar adecuadamente el debate liderado por Clara Campoamor en las Constituyentes de 1931.

\section{AGRADECIMIENTOS}

Este artículo se ha realizado en el marco del Proyecto I+D+i FEM2016-76675-P, Género, Compromiso y Transgresión en España, 1890-2016, MINECO, IP: Dra. Mónica Moreno Seco (Universidad de Alicante). Marta del Moral Vargas forma parte del Consejo del Instituto Universitario de Investigaciones Feministas, Universidad Complutense. Agradezco a Valentina Rodríguez y a María de la Concepción Pereira sus valiosos testimonios sobre Claudina y Luz García Pérez.

\section{NOTAS}

[1] Archivo de Villa de Madrid (AVM). Padrón Municipal de Habitantes, 19151930.

[2] AFSM, Actas asambleas y juntas directivas, FPI, Archivo y Biblioteca.

\section{BIBLIOGRAFÍA}

Agrupación Femenina Socialista de Madrid (1924, 26 de julio). A las Agrupaciones Femeninas Socialistas de España. El Socialista, p. 2.

Almond, G. y Verba, S. (1970). La cultura cívica: estudio sobre la participación política democrática en cinco naciones. Madrid: Euroamérica.

Aniversario de la muerte de Iglesias (1926, 8 de diciembre). La Voz, p. 2.

Arce Pinedo, R. (2008). Dios, Patria y Hogar. La construcción social de la mujer española por el catolicismo y las derechas en el primer tercio del siglo XX. Santander: Universidad de Cantabria.

Boxer, M. J. (2008). Repensar la construcción socialista y la posterior trayectoria internacional del concepto "Feminismo burgués”. Historia Social, 60, pp. 27-58.
[3] AFSM, Actas asambleas, (3-III-1912/18IX-1926), FPI, Archivo y Biblioteca, 25 de enero de 1925.

[4] AFSM, Actas de Asambleas, 17 de enero de 1926. Archivo del Congreso de

Campoamor, C. (1936/2001). El voto femenino y yo: mi pecado mortal. Madrid: Horas y Horas.

Canal, J. (1997). La Gran Familia. Estructuras e imágenes familiares en la base de la pervivencia del Carlismo. En: Cruz, R. y Pérez-Ledesma, M. (eds.). Cultura y movilización en la España contemporánea. Madrid: Alianza, pp. 99-136.

Capel Martínez, R. M. (1980). La mujer y el sindicalismo católico en la España de Alfonso XIII. Estudios de Historia Moderna y Contemporánea, 28 (116), pp. 355-375.

Capel Martínez, R. M. (1986). El trabajo y la educación de la mujer en España (19001930). Madrid: Instituto de la Mujer.

Círculo Socialista del Sur (1919, 7 de febrero). El País. Diario republicano progresista, p. 4. los Diputados. A las Cortes Constituyentes. Las mujeres españolas, Madrid, 9 de abril de 1932.

Fagoaga, C. (1985). La voz y el voto de las mujeres. El sufragismo en España, 18771931. Barcelona: Icaria.

Franco Rubio, G. (1981). La incorporación de la mujer a la Administración del Estado, Municipios y Diputaciones: 1918-1936. Madrid: Ministerio de Cultura.

García, C. y Hernández, M. (1921, 15 de abril). A los Grupos Femeninos de provincias. La Voz, p. 3.

García Basauri, M. (1979). La mujer "social", beneficencia y caridad en la crisis de la restauración. Tiempo de Historia, 59, pp. 28-31.

García Basauri, M. (1982). Los sindicatos católicos femeninos (1900-1930). Historia 16, 69, pp. 19-30. 
García Pérez, C. (1918, 28 de noviembre). Sólo tres mujeres. El Socialista, p. 1.

García Pérez, C. (1919, 20 de febrero). Las mujeres españolas. El Socialista, p. 2.

García Pérez, C. y García Pérez, L. (1928, 11 de noviembre). La educación de las mujeres. El Socialista, p. 2.

Gómez-Ferrer, G. y Moral Vargas, M. de (2015). Las pioneras en la gestión local: concejalas y alcaldesas designadas durante la dictadura de Primo de Rivera y el Gobierno Berenguer (1924-1930). En: Niefa Cristóbal, G. (coord.). Mujeres en los Gobiernos Locales. Alcaldesas y concejalas en la España contemporánea. Madrid: Biblioteca Nueva, pp. 41-71.

Gómez Llorente, L. (2004). Gestación y desarrollo del PSOE. En: PSOE 125. 125 años del Partido Socialista Obrero Español. Madrid: Fundación Pablo Iglesias, pp. 30-65.

Hernando Gonzalo, A. (2012). La fantasía de la individualidad. Buenos Aires: Katz. https://doi.org/10.2307/j.ctvm7bdns

Instituto de Reformas Sociales (1918). Preparación de un proyecto de Ley sobre el trabajo a domicilio. Madrid: Instituto de Reformas Sociales.

Juliá, S. (1996). Los socialistas en la política española, 1879-1982. Madrid: Taurus.

La Agrupación Socialista Madrileña y el voto femenino (1924, 24 de septiembre). La Libertad, p. 7.

La investigación de la paternidad (1926, 1 de mayo). El Liberal, p. 2.

Martín-Nájera, A. (dir.) (2010). Diccionario biográfico del socialismo español. [En línea]. Disponible en: http://www. fpabloiglesias.es/archivo-y-biblioteca/ diccionario-biografico

Meaker, G. H. (1978). La izquierda revolucionaria en España (1914-1923). Barcelona: Ariel.

Ministerio de Trabajo, Comercio e Industria. Dirección General de Trabajo y Acción Social (1927). Estadística de los salarios y jornadas de trabajo referida al periodo 1914 1925. Madrid: Ministerio de Trabajo.
Miralles Climent, J. (2005). Aspectos de la cultura política del carlismo en el siglo XX. Espacio, Tiempo y Forma. Serie V, Historia Contemporánea, 17, pp. 147-174. https:// doi.org/10.5944/etfv.17.2005.3119

Moral Sandoval, E (coord.), (2008). Centenario de la Casa del Pueblo de Madrid. Madrid: Sociedad Estatal de Conmemoraciones Culturas, UGT y Fundación Progreso y Cultura.

Moral Vargas, M. del (2012). Acción colectiva femenina en Madrid (1909-1931). Santiago de Compostela: Universidad de Santiago de Compostela.

Moral Vargas, M. del (2013). La Sociedad de Profesiones y Oficios Varios: un vivero para el sindicalismo madrileño (18941915). En: Castillo, S. (coord.). Mundo del trabajo y asociacionismo en España. Collegia, gremios, mutuas, sindicatos... Madrid: Catarata, pp. 1-21.

Movimiento obrero. Conferencia (1924, 20 de agosto). La Libertad, p. 6.

Movimiento Obrero. Fiesta de aniversario (1926, 11 de julio). La Libertad, p. 6.

Nash, M. (1996). Trabajadoras y estrategias de supervivencia económica: el caso del trabajo a domicilio. En: Vara Miranda, M. J. y Maquiera D’Angelo, V. (coords.). EI trabajo de las mujeres, siglos XVI-XX. VI Jornadas de Investigación Interdisciplinaria sobre la Mujer. Madrid: Universidad Autónoma de Madrid, pp. 355-366.

Natera Peral, A. (2001). El liderazgo político en la sociedad democrática. Madrid: Centro de Estudios Políticos y Constitucionales.

Nielfa Cristóbal, G. (1999). Mujeres: inmigración y trabajo en Madrid a lo largo del siglo XX. En: Aguado, A. (coord.). Las mujeres entre la historia y la sociedad contemporánea. Valencia: Generalitat Valenciana, Consellería de Bienestar Social y Universidad Internacional Menéndez Pelayo, pp. 135-163.

Notas societarias. Reunión del pleno nacional de Juventudes Socialistas (1925, 11 de noviembre). El Liberal, p. 2.

Núñez Orgaz, A. (1989). Las "modistillas" de Madrid, tradición y realidad (18841920). En: Bahamonde Magro, A. y Ote- ro Carvajal, L. E. (coords.). La sociedad madrileña durante la Restauración, 1876-1931, III Coloquio de Historia madrileña. Madrid: Comunidad Autónoma de Madrid, pp. 435-450.

Ortiz Albear, N. (2005). Las mujeres en la masonería. Málaga: Universidad de Málaga.

Radcliff, P. (1994). Política y cultura republicana en el Gijón de fin de siglo. En: Townson, N. (ed.). El republicanismo en España (1830-1977). Madrid: Alianza, pp. 373-394.

Ramos Palomo, M. D. (1998). Un compás para trazar una sociedad igualitaria: la labor de la librepensadora Belén Sárraga entre 1897 y 1909. Asparkía, 9, pp. 79-94.

Reuniones y conferencias. De Madrid. Círculo Socialista del Sur (1919, 7 de febrero). La Acción, p. 3.

Rey Reguillo, F. del (1992). Propietarios y patronos. La política de las organizaciones económicas en la España de la Restauración (1914-1923). Madrid: Ministerio de Trabajo y Seguridad Social.

Sanfeliu Gimeno, L. (2005a). Republicanas: identidades de género en el blasquismo. Valencia: Universitat de València.

Sanfeliu Gimeno, L. (2005b). Familias republicanas e identidades femeninas en el blasquismo: 1896-1910. Ayer, 60 (4), pp. 75-103.

Sanfeliu Gimeno, L. (2014). Educando para la democracia: las agrupaciones femeninas republicanas en la II República. En: Aguado Higón, A. y Sanfeliú Gimeno, L. (eds.), Caminos de democracia. Ciudadanía y culturas democráticas en el siglo XX. Granada: Comares, pp.225-241.

Ullman, J. (1972). La Semana Trágica. Estudio sobre las causas socioeconómicas del anticlericalismo en España (1898-1912). Barcelona: Ariel.

\section{Recursos en línea}

Presidencia del Directorio Militar. Estatuto Municipal de 8 de marzo de 1924. Gaceta de Madrid, 69, de 9 de marzo de 1924, pp. 1218-1302. [En línea]. Disponible en https://www.boe.es/datos/pdfs/ BOE//1924/069/A01218-01302.pdf 